\title{
Process evaluation in complex public health intervention studies: the need for guidance
}

\author{
Graham Moore, ${ }^{1}$ Suzanne Audrey, ${ }^{2}$ Mary Barker, ${ }^{3}$ Lyndal Bond, ${ }_{1}^{4}$ \\ Chris Bonell, ${ }^{5}$ Cyrus Cooper, ${ }^{3}$ Wendy Hardeman, ${ }^{6}$ Laurence Moore, ${ }^{7,8}$ \\ Alicia O'Cathain, ${ }^{9}$ Tannaze Tinati, ${ }^{3}$ Danny Wight, ${ }^{10}$ Janis Baird ${ }^{3}$
}

\section{WHY IS PROCESS EVALUATION NEEDED IN COMPLEX PUBLIC HEALTH INTERVENTION RESEARCH?}

Public health interventions aim to improve the health of populations or at-risk subgroups. Problems targeted by such interventions, such as diet and smoking, involve complex multifactorial aetiology. Interventions will often aim to address more than one cause simultaneously, targeting factors at multiple levels (eg, individual, interpersonal, organisational), and comprising several components which interact to affect more than one outcome. ${ }^{1}$ They will often be delivered in systems which respond in unpredictable ways to the new intervention. ${ }^{2}$ Recognition is growing that evaluations need to understand this complexity if they are to inform future intervention development, or efforts to apply the same intervention in another setting or population. ${ }^{1}$ Achieving this will require evaluators to move beyond a 'does it work?' focus, towards combining outcomes and process evaluation. There is no such thing as a typical process evaluation, with the term applied to studies which range from a few simple quantitative items on satisfaction, to complex mixed-method studies exploring issues such as the process of implementation, or contextual influences

${ }^{1}$ DECIPHer UKCRC Public Health Research Centre of Excellence, School of Social Sciences, Cardiff University, Cardiff, UK; ${ }^{2}$ DECIPHer UKCRC Public Health Research Centre of Excellence, School of Social and Community Medicine, University of Bristol, Bristol, UK; ${ }^{3} \mathrm{MRC}$ Lifecourse Epidemiology Unit, University of Southampton, Southampton, UK; ${ }^{4}$ Centre of Excellence in Intervention and Prevention Science, Melbourne, VIC Australia; ${ }^{5}$ Social Science Research Unit, Institute of Education, University of London, London, UK; ${ }^{6}$ The Primary Care Unit, Department of Public Health and Primary Care, University of Cambridge, Cambridge, UK; ${ }^{7}$ Cardiff University Schools of Social Sciences and Medicine, Cardiff, UK; ${ }^{8}$ DECIPHer UKCRC Public Health Research Centre of Excellence, Cardiff, UK; ${ }^{9}$ School of Health and Related Research, University of Sheffield, Sheffield, UK; ${ }^{10}$ MRC Social and Public Health Sciences Unit, GLASGOW, UK

Correspondence to Dr Graham Moore, DECIPHer UKCRC Public Health Research Centre of Excellence, School of Social Sciences, Cardiff University, Cardiff cf103bd, UK; MooreG@cardiff.ac.uk on implementation and outcomes. As recognised within MRC guidance for evaluating complex interventions, process evaluation may be used to 'assess fidelity and quality of implementation, clarify causal mechanisms and identify contextual factors associated with variation in outcomes'. ${ }^{1}$ This paper briefly discusses each of these core aims for process evaluation, before describing current Medical Research Council (MRC) Population Health Sciences Research Network (PHSRN) funded work to develop guidance for process evaluations of complex public health interventions.

\section{Intervention implementation}

An important role for process evaluations is to examine the quantity and quality of what was actually implemented in practice, and why. This may inform implementation of similar interventions elsewhere, and facilitate interpretation of intervention outcomes. While notions of standardisation are central to implementation assessment, the nature of standardisation required in complex interventions is debated. Hawe and colleagues argue that tailoring intervention from across contexts may ensure greater consistency with intended functions. ${ }^{3}$ Others argue that when we know little about which are core active ingredients, we might be tempted to make adaptations which inhibit effectiveness. ${ }^{4}$ Durlak and DuPre argue that 'core components should receive emphasis in terms of fidelity', with remaining components allowed to vary. ${ }^{5}$ All these positions highlight the need for implementation assessments within process evaluations to be guided by a thorough knowledge of the intervention theory, in terms of which components are expected to do what, and how changes during implementation may impede or optimise functioning. While assessments of feasibility will likely be made in exploratory evaluation phases, new challenges in implementation may need to be understood as an intervention moves into a wider range of contexts for a larger scale evaluation of effectiveness. Exploring issues such as acceptability may also enable evaluators to better understand intervention delivery and reach.

\section{Causal mechanisms}

All public health interventions reflect implicit or explicit theories regarding how a course of action will solve a perceived problem. However, the contribution of outcomes evaluations to theory development is often limited by failures to clearly articulate the assumptions being made, as well as naive tendencies to presume that what was implemented was consistent with these assumptions, as discussed above. MRC guidance argues that only through close scrutiny of causal mechanisms is it possible for evaluation to contribute to developing more effective interventions, and provide insights into how findings might be transferred across settings and populations. ${ }^{1}$ Some early frameworks for process evaluations in public health focused predominantly on implementation and context, paying more limited attention to causal mechanisms. ${ }^{6}$ However, the need for evaluations to test and develop intervention theory, rather than simply provide pass or fail assessments of effectiveness, is recognised within approaches such as Realistic Evaluation, ${ }^{7}$ the more recently proposed concept of Realist Trials, ${ }^{8}$ and recent recommendations for process evaluation within cluster randomised trials. ${ }^{9}$ Process evaluations may usefully combine quantitative assessments of hypothesised mediators with qualitative investigation of unanticipated or complex causal pathways, and unanticipated consequences.

\section{Contextual factors}

Understanding the contexts in which complex interventions are delivered and received is critical to explaining why they do or do not work, or how we might expect impacts to differ if implemented elsewhere. 'Context' may include any factors which are external to the intervention, but which may impede or strengthen the effects of an intervention. This may include factors which existed prior to the intervention, or factors which emerge during its implementation. While early process evaluation frameworks emphasised roles of context in shaping implementation, ${ }^{6}$ contextual factors may also moderate outcomes. Implementation will often require complex behavioural changes among implementers, and may be influenced by factors such as their circumstances, skills and attitudes. ${ }^{7} \quad 10 \quad 11$ Similarly, participants do not receive interventions passively but interact with them in ways influenced by their circumstances, 
attitudes, beliefs, social norms and resources. The causal pathways of problems targeted by public health intervention will differ from one time and place to another. Hence, the same intervention may have different consequences if implemented in a different setting, or among different subgroups. Even where an intervention itself is relatively simple, its causal processes and relationship with its context may still be considered complex.

\section{WHY IS GUIDANCE NEEDED?}

In 2008 the MRC updated their guidance on the development and evaluation of complex interventions. As described above, in a change in emphasis from the earlier version published in 2000, revised guidance called for definitive evaluation to combine evaluation of outcomes with that of process. ${ }^{1}$ It did not, however, offer detail on how to conduct process evaluation. Existing frameworks offer some assistance to researchers conducting process evaluations. ${ }^{6}$ However, these tend to focus on identifying priority aims for process evaluations, rather than providing detailed guidance on how to plan, design or conduct a process evaluation.

The shortage of guidance to assist researchers, grant referees or journal reviewers in the design, conduct or assessment of process evaluation means that process evaluation may be planned in an ad hoc fashion. Hence, while process evaluation is an increasingly common component of grant applications, study protocols and published evaluations of public health interventions approaches are highly variable. ${ }^{9}$ The appearance of variability in approaches is perhaps heightened further by inconsistency in reporting, perhaps stemming from challenges reporting process data in a single article while remaining within journal word limits. Furthermore, there is often limited integration between process and outcomes studies. $^{12}$

\section{DEVELOPING GUIDANCE FOR PROCESS EVALUATIONS OF COMPLEX PUBLIC HEALTH INTERVENTIONS}

In November 2010 an MRC PHSRNfunded workshop discussed the need for guidance on process evaluation in complex public health intervention studies. The workshop was conceived by a number of groups undertaking complex intervention research that had identified the need to draw on collective expertise in developing process evaluation. Workshop participants, who were predominantly public health researchers and policy makers, were strongly in favour of the development of guidance to assist them in their research. There was also consensus that funders and reviewers of grant applications would benefit from guidance to assist peer review.

Following the workshop, the MRC PHSRN is supporting development of guidance for process evaluation of complex public health interventions, focusing predominantly upon process evaluations within trials or other outcomes evaluations. The authors form the group leading the development of guidance. It will focus upon priorities for process evaluation identified within the MRC framework for complex interventions, in terms of understanding implementation, causal mechanisms and the relationship of interventions with their contexts. The guidance is being developed through an iterative process of literature review, reflection on detailed case studies of process evaluations in which the guideline development group has been involved, and consultation with stakeholders including researchers, journal editors, and policy and practice partners. The aim of this endeavour is to provide public health researchers, policy makers, research funders and journal editors with guidance in designing, conducting and appraising process evaluations of complex public health interventions.

Contributors GM and JB are leading the work. All authors form a guideline development group who have discussed and agreed the scope of the work, and the approaches to be taken, and all of whom have provided comments on earlier drafts of the manuscript.

Funding The work is funded by the MRC Population Health Sciences Research Network. The work is undertaken with the support of The Centre for the Development and Evaluation of Complex Interventions for Public Health Improvement (DECIPHer), a UKCRC Public Health Research: Centre of Excellence. Funding from the British Heart Foundation, Cancer Research UK, Economic and Social Research Council (RES-590-28-0005), Medical Research Council (PHSRN45), the Welsh Government and the Wellcome Trust (WT087640MA), under the auspices of the UK Clinical Research Collaboration, is gratefully acknowledged.

\section{Competing interests None.}

Provenance and peer review Not commissioned; externally peer reviewed.

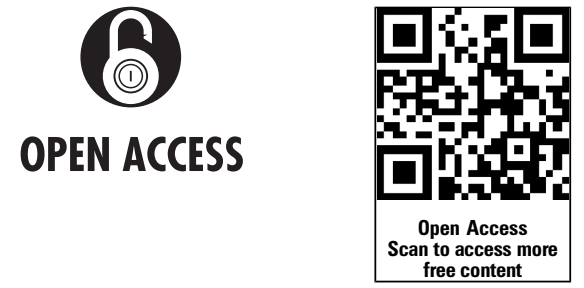

Open Access This is an Open Access article distributed in accordance with the Creative Commons Attribution (CC BY 3.0) license, which permits others to distribute, remix, adapt, build upon this work, for commercial use, provided the original work is properly cited. See: http:// creativecommons.org/licenses/by/3.0/

To cite Moore G, Audrey S, Barker M, et al. J Epidemiol Community Health 2014;68:101-102.

Received 15 May 2013

Revised 30 July 2013

Accepted 13 August 2013

Published Online First 10 September 2013

J Epidemiol Community Health 2014;68:101-102. doi:10.1136/jech-2013-202869

\section{REFERENCES}

1 Craig P, Dieppe P, Macintyre S, et al. Developing and evaluating complex interventions: the new Medical Research Council guidance. BMJ 2008;337:a1655.

2 Keshavarz N, Nutbeam D, Rowling L, et al. (2010) Schools as social complex adaptive systems: a new way to understand the challenges of introducing the health promoting schools concept. Soc Sci Med 2010;70:1467

3 Hawe P, Shiell A, Riley T. Complex interventions: how out of control can a randomised controlled trial be? BMJ 2004:328:1561-3.

4 Mihalic $S$. The importance of implementation fidelity. Emo Behav Disorders Youth 2004;4:83-6.

5 Durlak JA, DuPre EP. Implementation matters: A review of research on the influence of implementation on program outcomes and the factors affecting implementation. Am J Community Psychol 2008:41:327-50.

6 Steckler A, Linnan L. eds. Process evaluation for public health interventions and research. San Francisco: Jossey-Bass, 2002.

7 Pawson R, Tilley N. Realistic evaluation. London: Sage, 1997

8 Bonell C, Fletcher A, Morton M, et al. Realist randomised controlled trials: a new approach to evaluating complex public health interventions. Soc Sci Med. 2012;75:2299-306.

9 Grant A, Treweek S, Dreischulte T, et al. Process evaluations for cluster-randomised trials of complex interventions: a proposed framework for design and reporting. Trials 2013;14:15.

10 Berwick DM. The science of improvement. J Amer Med Assoc. 2008;299:1182-4.

11 Glasgow RE, Lichtenstein E, Marcus AC. Why don't we see more translation of health promotion research to practice? Rethinking the efficacy-to-effectiveness transition. Am J Public Health 2003;93:1261-7.

12 Lewin S, Glenton C, Oxman AD. Use of qualitative methods alongside randomised controlled trials of complex healthcare interventions: methodological study. BMJ 2009;339:b3496. 


\section{Corrections}

Moore G, Audrey S, Barker M, et al. Process evaluation in complex public health intervention studies: the need for guidance. J Epidemiol Community Health 2014;68:101-2. The Open Access licence should be CC-BY.

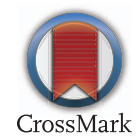

J Epidemiol Community Health 2014;68:585. doi:10.1136/jech-2013-202869corr1 